\title{
Bildungsbegleitung für Familien
}

\section{Sechs Wahrheiten über Chancengleichheit und frühe Bildung}

\section{ANGELA SCHRÖDER,}

DETLEF KRÜGER UND

REINHARD KAUT

Dr. Angela Schröder ist Geschäftsführerin der healthcompany - Institut für angewandte Gesundheitsforschung $\mathrm{GmbH}$ in Hamburg. Sie hat Gesundheitswissenschaften studiert und an der Universität Dresden zum Dr. rer. medic promoviert. Ihre Arbeitsschwerpunkte sind Prävention, Gesundheitsbildung, Coaching und Prozessbegleitung, Weiterbildung und Training, Projektevaluation sowie Mitarbeit bei der Konzeptentwicklung des Bundesprogramms "Elternchance ist Kinderchance".

www.healthcompany-hamburg.de/

Prof. Dr. Detlef Krüger ist Hochschullehrer für Sozialwissenschaften; er lehrt an der HAW Hamburg. Seine Forschungsschwerpunkte sind Kindergesundheit, Familienentwicklung, Human Ressource Management, eLearning. Seine Tätigkeiten in der Praxis: Gesundheitsförderung, Familienbildung, Moderation und Evaluation von Veränderungsprozessen, Er hat bei der Konzeptentwicklung des Bundesprogramms "Elternchance ist Kinderchance" mitgearbeitet.

www.haw-hamburg.de

Reinhard Kaut ist Geschäftsführer der Bundesarbeitsgemeinschaft für Familienbildung und Beratung e. V. (BAG) in Elmshorn. Er ist seit vielen Jahren in der Familienbildung tätig. Sein aktueller Arbeitsschwerpunkt ist die Mitgestaltung des Konzepts und die Umsetzung des Bundesprogramms "Elternchance ist Kinderchance« des Bundesministeriums für Familie, Senioren, Frauen und Jugend. www.familienbildung.de

\author{
Das finanzielle Engagement bei der frühen Förderung \\ von Kindern in Kitas und im Vorschulbereich ist in \\ Deutschland als zu gering einzuschätzen. Die fehlenden \\ Mittel schlagen sich in für Kinder nachteiligen \\ Personalschlüsseln für die Betreuung, Erziehung und \\ Bildung in allen Institutionen bis zum Schuleintritt sowie \\ in der schlechten tariflichen Bezahlung der Beschäftigten \\ nieder. Diese Situation erfordert sowohl einen \\ Politikwechsel als auch eine Umkehr im Denken über die \\ Wertigkeit von Kindern als gesellschaftliche Aufgabe.
}

In den modernen Informations- und Dienstleistungsgesellschaften erlangen die Fähigkeiten und Kenntnisse des Einzelnen immer mehr an Bedeutung, wodurch Bildung zunehmend eine entscheidende Voraussetzung für gesellschaftliche Teilhabe von Menschen darstellt. Der Bildungserfolg im Kindesund Jugendalter hat weitreichende Konsequenzen für die Berufs- und Lebenschancen als Erwachsener. Gleichzeitig gilt die Gleichheit oder Angleichung von Bildungschancen als besonders wichtiges gesellschaftspolitisches Ziel.

Die zunehmende Bedeutung von Bildung findet ihren Ausdruck nicht zuletzt in der sogenannten Bildungsexpansion. Auch wenn in den letzten Jahrzehnten insgesamt alle Bevölkerungsschichten von der Bildungsexpansion profitiert haben, sind die Bildungsungleichheiten nach der sozialen Herkunft in Deutschland nach wie vor sehr ausgeprägt. Die Bildungschancen von Kindern sind umso größer, je höher das Bildungsniveau ihrer Eltern ist. Wirken vorteilhafte oder nachteilige Berufsstellungen, Bildungsgrade und Einkommensverhältnisse der Eltern zusammen, ergeben sich besonders gute oder besonders schlechte Bildungschancen für die Kinder. Aufgrund der sozialen Selektivität des deutschen Bildungssystems werden insbesondere
Kinder aus »bildungsfernen « Familien zu Bildungsverlierern (vgl. Ditton/Krüsken 2009, Becker/Lauterbach 2012).

Bildungsferne Familien sind in der Regel dadurch gekennzeichnet, dass sie aufgrund ihres »kulturellen « Kapitals keinen oder nur einen erschwerten Zugang zum deutschen Bildungssystem finden. Vor dem Hintergrund eigener Bildungserfahrungen, des eigenen erreichten Bildungsabschlusses und der Abschätzung eigener Unterstützungsmöglichkeiten für den Bildungsweg der Kinder wird Bildung unterschiedlich bewertet und entsprechend orientieren sich die Bildungsentscheidungen der Eltern häufig nicht an den Entwicklungspotenzialen der Kinder. Erschwerend kommt für bildungsungewohnte Familien hinzu, dass die deutsche Schule auch heute noch überwiegend eine "Mittelklasseinstitution " darstellt, die den kulturellen Normen, Werten und Symbolen der Bildungsschichten entspricht. Insofern werden Schullaufbahnempfehlungen seitens der Schule vielfach eher von der sozialen Herkunft als vom Leistungsvermögen eines Kindes bestimmt.

Um mehr Offenheit in Bildungsverläufen zu erreichen, sind insbesondere in bildungsfernen Familien eine wirksame Aufklärung über Bildung, Bildungsverläufe und soziale Mobilität notwendig. Im Zuge einer Intensivierung frühkindlicher 
Bildung und Betreuung können vor allem Kinder aus sozial benachteiligten Familien und aus Familien mit Migrationshintergrund von infrastruktureller Familienberatung und Begleitung profitieren.

Bildungsbegleitung als Elternberatung hat sich um den generellen Stellenwert von Bildung in Familien zu bemühen sowie Eltern hinsichtlich der Bildungsübergänge ihrer Kinder zu unterstützen. Bildungsbegleitung sollte bei allen Maßnahmen immer das Ziel verfolgen: »Eltern stärken«.

Bildungsübergänge von Kindern sind in Familien häufig auch kritische Phasen in der Familienentwicklung. Eltern sind für zahlreiche Bildungsentscheidungen in Form der Auswahl spezifischer Lernorte und Lernformen ihrer Kinder verantwortlich. So entscheiden sie sich beispielsweise für den Besuch einer Kindertageseinrichtung je nach Alter des Kindes, Dauer der täglichen Betreuung und inhaltlichen Förderungskonzepten der Einrichtung. Oder Eltern bestimmen den Übergang ihrer Kinder in eine bestimmte Schulform nach Abschluss der Grundschule. Dabei mangelt es bislang erheblich daran, diese Übergänge als Bedarfssituationen von Familien zu erkennen sowie in diesem Zusammenhang ziel- und zeitgerechte Angebote für Familien als Beratung zur Verfügung zu stellen.

Nach dem Konzept der Entwicklungsaufgaben gibt es für eine gesunde körperliche, geistige und psychische Entwicklung eines Kindes eine relativ klare Zeitstruktur für notwendige Entwicklungsschritte im Lebensverlauf (Piaget, Vygotzky, Erikson, Havighurst, Kohlberg). Den Entwicklungsprozessen des Kindes stehen dabei komplementäre Entwicklungsaufgaben der Eltern gegenüber (Bowlby, Ainsworth, Bronfenbrenner).

Aufbauend auf diesen Erkenntnissen wurde in der neueren bildungsökonomischen Forschung die Entwicklung von Kompetenzen und Fähigkeiten im Lebensverlauf als dynamischer Prozess modelliert (Cunha et al. 2006). Nach dem Modell dynamischer Kompetenzentwicklung (»skill formation«) können folgende weltweit empirisch belegte sechs Aussagen für Bildungsprozesse getroffen werden:

\footnotetext{
1 Die Entwicklung und Förderung - der Fähigkeiten von Kindern werden schon in einem frühen Alter als herkunftsabhängige Differenzierungen beobachtet.
}

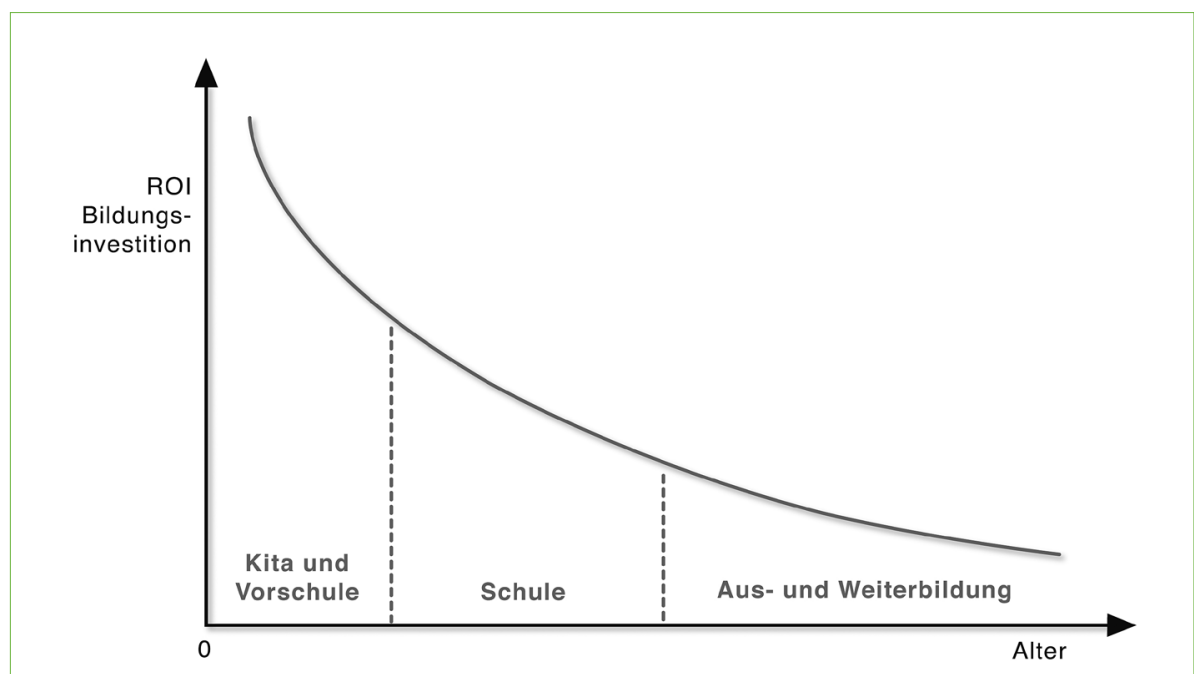

Je früher Bildungsinvestitionen getätigt werden, desto größer ist ihr Erfolg - mit zunehmendem Alter nimmt der ökonomische Nutzen von Bildungsinvestitionen ab.

Kompetenzen werden bei Betrach- tung der Lernfortschritte innerhalb spezifischer Entwicklungsstufen optimal herausgebildet.

3. Eine präventive Entwicklungs- förderung von herkunftsbezogen schlechter gestellten Kindern in Institutionen früher Bildung zeichnet sich durch einen hohen Erfolg aus.

Die frühe Förderung erzielt durch - wiederholende Maßnahmen späterer Förderung eine höhere Wirksamkeit (Nachhaltigkeit).

5 Finanzielle Einschränkungen bei - Bildungsinvestitionen sind in der frühen Kindheit für den Bildungsverlauf im Ergebnis negativer als in jugendlichen Entwicklungsphasen.

Sozial-emotionale Kompetenzen - fördern kognitive Lernprozesse und sind in dieser Hinsicht - vor allem in der frühen Kindheit - ein wesentlicher Faktor für positive Bildungsverläufe.

Auf Basis des Modells dynamischer Kompetenzentwicklung haben Heckman und Cunha eine Human-CapitalInvestment-Funktion angegeben, die den Return-on-Investment (ROI) über alle Altersstufen bei einem vorgegebenen gleichen Betrag einer Bildungsinvestition beschreibt (vgl. Abbildung). Die dargestellte Funktion basiert auf Ergebnissen einer Vielzahl weltweit verfügbarer empirischer Studien. Mit dieser Funktion wird der dynamische Charakter der Entwick- lung des Humankapitals über den Lebensverlauf sichtbar - mit zunehmendem Alter nimmt der ökonomische Nutzen von Bildungsinvestitionen ab. Ein hoher Return-on-Investment wird vor allem im Kita-Alter von Kindern erreicht. Insofern sind Bildungschancen in der Familie und in Institutionen früher Förderung nicht nur die wesentlichen Voraussetzungen für einen gelungenen Bildungsverlauf von Kindern, sondern auch Richtschnur für eine effektive Bildungspolitik.

Es hat sich empirisch immer wieder gezeigt, dass insbesondere Kinder aus benachteiligten Familien von einer frühzeitigen institutionellen Förderung profitieren und insofern die Möglichkeiten einer kompensatorischen Erziehung und Bildung für Kinder außerhalb der Familie bis zum Erreichen des Schulalters besonders wirksam sind (vgl. Heckman 2013).

Das dargestellte bildungsökonomische Modell lässt erhebliche Zweifel für eine Bildungspolitik aufkommen, die diesen ökonomischen Gesetzmäßigkeiten in keiner Weise Rechnung trägt. So sind die deutschen Bildungsanstrengungen früher Förderung von Kindern in Kitas und im Vorschulbereich hinsichtlich der finanziellen Ausstattung als defizitär zu betrachten. Die geringe Finanzierungsgrundlage schlägt sich in für Kinder nachteiligen Personalschlüsseln für die Betreuung, Erziehung und Bildung in allen Institutionen bis zum Schuleintritt sowie in der schlechten tariflichen Bezahlung der dortigen Beschäftigten nieder. Der nur noch durchschnittliche Bildungserfolg im schulischen und universitären Bereich in Deutschland ist nicht zuletzt 
ein Ergebnis der seit vielen Jahren weit unter dem OECD-Durchschnitt liegenden Bildungsausgaben im Kita- und Vorschulbereich (OECD 2006). Die Verbesserung dieser Situation erfordert nicht nur einen Politikwechsel, sondern auch eine Umkehr im Denken über die Wertigkeit von Kindern als gesellschaftliche Aufgabe. Insofern steht in Deutschland für die Bildungssituation von Kindern im Kita- und Vorschulalter eine Exzellenzinitiative "Kita « mit entsprechender finanzieller Förderung für Infrastruktur und Personalausstattung aus.

\section{Bildungsberatung für Familien} katoren feststellen, dass es in Deutschland eine hohe Zahl von Bildungsverlierern gibt. Einen überaus großen Bereich von Verlierern stellt die Gruppe der bildungsungewohnten Familien dar, die daneben sehr häufig auch zur Gruppe der sozio-ökonomisch Benachteiligten gehört. Defizite tun sich insbesondere dort auf, wo es nicht gelingt, Eltern frühzeitig und als wesentliche Zielgruppe für Bildungsberatung zu erreichen. Insofern sind große Anstrengungen notwendig, um mit einer niedrigschwelligen Eltern-
Wir müssen auf Basis von Bildungsindi-

Curriculum fokussiert auf den Erwerb kommunikationsbezogener, beratungstechnischer und bildungssoziologischer Qualifikationen im Hinblick auf die Stärkung von Kompetenzen der Eltern. Die Zertifizierungsmaßnahme richtet sich vor allem an Fachkräfte in Familienzentren, Einrichtungen der Familienbildung, Eltern-Kind-Zentren, Kindertageseinrichtungen und Mehrgenerationenhäuser. Mit diesem Angebot wird ein sozialraumorientiertes, niedrigschwelliges Bildungs- und Beratungskonzept für die Praxis zur Verfügung gestellt.

Mit dem Bundesprogramm »Elternchance ist Kinderchance" entsteht die Möglichkeit, den angesprochenen Familienkreis in einer Breitenwirkung zu erreichen (BMFSFJ 2013). Neben der Qualifizierung von 4.000 Fachkräften für Familienarbeit im Hinblick auf Elternberatung und Bildungsbegleitung von Kindern wird die Intensität der Vernetzung zu den wesentlichen $\mathrm{Zu}$ gangswegen der Elternerreichbarkeit in 100 Modellstandorten erprobt: Hier sind vor allem auch Familienbildungsstätten, Eltern-Kind-Zentren, FamilienZentren, Kindertagesstätten, Elternschulen, Mehrgenerationenhäuser im Blick des Bundesprogramms.

\section{"Bildungsbegleitung sollte vor allem ein Ziel haben: Eltern zu stärken«}

beratung eine Vielzahl von Familien für die Bildungsverläufe ihrer Kinder zu interessieren, zu motivieren und zu stärken.

Hinsichtlich dieser Zukunftsaufgabe der Eltern- und Familienbildung wurde von der Bundesarbeitsgemeinschaft Familienbildung und Beratung e. V. (BAG) im Rahmen der Förderung des Bundesprogramms »Elternchance ist Kinderchance e ein Curriculum für die zertifizierte Weiterbildung Elternberater und Elternbegleiter zu den Bildungsverläufen von Kindern konzipiert und umgesetzt (BAG 2013).

Die von der Bundesarbeitsgemeinschaft Familienbildung und Beratung angebotene Zertifizierungsmaßnahme qualifiziert Fachkräfte als Elternberater für die Beratung von bildungsungewohnten und benachteiligten Familien zu Bildungsprozessen und Bildungsentscheidungen in den Bildungsverläufen ihrer Kinder. Das handlungsorientierte
U3-Betreuung, Kita und Schule in ihrer gesunden Entwicklung im Blick haben.

Überall dort, wo Kommunen die Lebenslagen der Familien als Ansatz für die Förderung von Kindern betrachten, können Konzepte der Elternberatung zu den Bildungsverläufen von Kindern als weiteres Glied einer Präventionskette nachhaltig integriert werden. Mit dieser Herangehensweise werden sehr viel mehr Kinder aus benachteiligten Familien eine faire Chance im Bildungsverlauf bekommen.

\section{Literatur}

Barnett, M.S. (2004). Benefit-cost analysis of preeschool education. http://nieer.org/ resources/files/BarnettBenefits.ppt (Zugriff 30.11.2010).

BAG (Hg.) (2013). Elternbegleiter.

http://familienbildung.de/infos/ elternbegleiter (Zugriff 30.4.2013)

Becker, R., Lauterbach, W. (Hg.) (2012). Bildung als Privileg? Erklärungen und Befunde zu den Ursachen der Bildungsungleichheit. Wiesbaden: VS Verlag.

BMFSFJ (Hg.) (2013). Bundesprogramm »Elternchance ist Kinderchance". www.elternchance.de (Zugriff 30.4.2013).

Bronfenbrenner, U. (1974). Wie wirksam ist kompensatorische Erziehung? Stuttgart: Ernst Klett Verlag.

Cunha, F., Heckman, J. J., Lochner, L. L., Masterov, D. (2006). Interpreting the Evidendence on Life Cycle Skill Formation. In: Hanushek, E., Welch, F. (ed.). Handbook of the Economics of Education, 697-812. Amsterdam: North Holland.

Ditton, H., Krüsken, J. (2009). Bildungslaufbahnen im differenzierten Schulsystem. Entwicklungsverläufe von Laufbahnempfehlungen und Bildungsaspirationen in der Grundschulzeit. In: Baumert, J., Maaz, K., Trautwein, U. (Hg.). Bildungsentscheidungen. Zeitschrift für Erziehungswissenschaft, Sonderheft 12.

Fritschi, T., Oesch, T. (2008). Volkswirtschaftlicher Nutzen von frühkindlicher Bildung in Deutschland. www.bertelsmann-stiftung.de (Zugriff: 8.8.2010).

Heckman, J. J., Moon, S. H., Pinto, R., Savelyev, P.A., Yavitz, A.Q. (2010). The rate of return to the HighScope Perry Preschool Program. Journal of Public Economics 94, 1-2: 114-128. Heckman, J.J. (2013). Giving Kids A Fair Chance. Cambridge, Mass.: Boston Review Books.

OECD (Ed.) (2006). Starting Strong II. Paris: OECD. Tietze, W., Rossbach, H.-G., Grenner, K. (2005). Kinder von 4 bis 8 Jahren. Weinheim und Basel: Beltz Verlag. 